University of Nebraska - Lincoln

DigitalCommons@University of Nebraska - Lincoln

2012

\title{
Ultrahard magnetic nanostructures
}

\author{
P. K. Sahota \\ University of Nebraska-Lincoln
}

Yi Liu

University of Nebraska-Lincoln, yliu@unl.edu

Ralph Skomski

University of Nebraska-Lincoln, rskomski2@unl.edu

Priyanka Manchanda

LNM Institute of Information Technology, priyanka.manchanda@vanderbilt.edu

\author{
R. Zhang \\ University of Nebraska-Lincoln
}

See next page for additional authors

Follow this and additional works at: https://digitalcommons.unl.edu/physicssellmyer

Part of the Physics Commons

Sahota, P. K.; Liu, Yi; Skomski, Ralph; Manchanda, Priyanka; Zhang, R.; Franchin, M.; Fangohr, H.; Hadjipanayis, George C.; Kashyap, Arti; and Sellmyer, David J., "Ultrahard magnetic nanostructures" (2012). David Sellmyer Publications. 238.

https://digitalcommons.unl.edu/physicssellmyer/238

This Article is brought to you for free and open access by the Research Papers in Physics and Astronomy at DigitalCommons@University of Nebraska - Lincoln. It has been accepted for inclusion in David Sellmyer Publications by an authorized administrator of DigitalCommons@University of Nebraska - Lincoln. 


\section{Authors}

P. K. Sahota, Yi Liu, Ralph Skomski, Priyanka Manchanda, R. Zhang, M. Franchin, H. Fangohr, George C. Hadjipanayis, Arti Kashyap, and David J. Sellmyer 


\title{
Ultrahard magnetic nanostructures
}

\author{
P. K. Sahota, ${ }^{1,2}$ Y. Liu, ${ }^{1}$ R. Skomski, ${ }^{1}$ P. Manchanda, ${ }^{1,2}$ R. Zhang, ${ }^{1}$ M. Franchin, ${ }^{4}$ \\ H. Fangohr, ${ }^{4}$ G. C. Hadjipanayis, ${ }^{3}$ A. Kashyap, ${ }^{2, a)}$ and D. J. Sellmyer ${ }^{2}$ \\ ${ }^{1}$ Department of Physics and Astronomy and Nebraska Center for Materials and Nanoscience, \\ University of Nebraska, Lincoln, Nebraska 68588, USA \\ ${ }^{2}$ The LNM Institute of Information Technology Jaipur, Rajasthan 302031, India \\ ${ }^{3}$ Physics Department, University of Delaware, Newark, Delaware 19716, USA \\ ${ }^{4}$ Engineering and Environment, University of Southampton, Southampton SO17 1BJ, UK
}

(Presented 1 November 2011; received 23 September 2011; accepted 13 December 2011; published online 13 March 2012)

\begin{abstract}
The performance of hard-magnetic nanostructures is investigated by analyzing the size and geometry dependence of thin-film hysteresis loops. Compared to bulk magnets, weight and volume are much less important, but we find that the energy product remains the main figure of merit down to very small features sizes. However, hysteresis loops are much easier to control on small length scales, as epitomized by Fe-Co-Pt thin films with magnetizations of up to $1.78 \mathrm{~T}$ and coercivities of up to 2.52 T. Our numerical and analytical calculations show that the feature size and geometry have a big effect on the hysteresis loop. Layered soft regions, especially if they have a free surface, are more harmful to coercivity and energy product than spherical inclusions. In hard-soft nanocomposites, an additional complication is provided by the physical properties of the hard phases. For a given soft phase, the performance of a hard-soft composite is determined by the parameter $\left(\mathbf{M}_{\mathrm{s}}-\mathbf{M}_{\mathrm{h}}\right) / \mathrm{K}_{\mathrm{h}}$. (C) 2012 American Institute of Physics. [doi:10.1063/1.3679453]
\end{abstract}

\section{INTRODUCTION}

Permanent magnets are typically judged by the energy product, which is, basically, energy per unit volume of magnetic material. ${ }^{1,2}$ This consideration is important for bulk applications, for example, in cars where magnet weight and volume matter. However, the magnet volume is not the main consideration in small-scale nanostructures ${ }^{3}$ and in thin films for MEMS applications, and the question arises whether the energy product remains a valid figure of merit.

In this paper, we discuss alternative figures of merit, such as the hardness product, and answer the question which materials combinations and geometries are best suitable for certain permanent-magnet applications.

\section{NANOSCALE ENERGY PRODUCTS}

It is tempting to use the coercivity as a key figure of merit, particularly since the mass and volume of magnetic materials is less important in thin-film applications than in bulk magnets. However, the coercivity is roughly proportional to the anisotropy field $H_{\mathrm{A}}=2 K_{1} / \mu_{\mathrm{o}} M_{\mathrm{o}}$, where $K_{1}$ is the first anisotropy constant and $M_{\mathrm{o}}$ is the saturation magnetization. It is well-known that $H_{\mathrm{A}}$ can be made arbitrarily large by choosing nearly compensated ferrimagnets with $M_{\mathrm{o}} \approx 0$, but such materials do not create a magnetic field in free space and are not suitable for most hard-magnetic applications.

Another possible choice is the hardness product, defined as the product of coercivity and remanence. ${ }^{4}$ The hardness product includes the magnetization as a key requirement, but since $M_{\mathrm{r}} H_{\mathrm{c}}$ scales as $M_{\mathrm{o}} 2 K_{1} / \mu_{\mathrm{o}} M_{\mathrm{o}}$, it is essentially proportional

\footnotetext{
a) Author to whom correspondence should be addressed. Electronic mail: arti.kashyap@gmail.com.
}

to $K_{1}$. This overestimates the performance of highly coercive magnets with small magnetization.

A better approach is to request the stability of the magnetization in stray fields, which are proportional to the magnetization itself. This criterion is unrelated to the magnet volume, but it means excess coercivity beyond $M_{\mathrm{o}}$ does not further improve the magnets performance, very similar to the traditional energy product. We therefore advocate the use of $(\mathrm{BH})_{\max }$ as a figure of merit even in thin-film nanostructures, where the magnet volume is not a major consideration.

Some of these nanostructures are actually very hard, such as $L 1_{0}$-ordered Fe-Pt thin-film patches. ${ }^{5}$ Our Fe-Co-Pt thinfilm magnets have a thickness of $20 \mathrm{~nm}$ and roomtemperature properties of $\mu_{\mathrm{o}} H_{\mathrm{c}}=2.52 \mathrm{~T}, \mu_{\mathrm{o}} M_{\mathrm{s}}=1.67 \mathrm{~T}$ and $(B H)_{\max }=444 \mathrm{~kJ} / \mathrm{m}^{3}$ for $\mathrm{Fe}_{39} \mathrm{Co}_{21} \mathrm{Pt}_{40}$, and $\mu_{\mathrm{o}} H_{\mathrm{c}}=0.89 \mathrm{~T}$, $\mu_{\mathrm{o}} M_{\mathrm{s}}=1.78 \mathrm{~T}$ and $(B H)_{\max }=465 \mathrm{~kJ} / \mathrm{m}^{3}$ for $\mathrm{Fe}_{41} \mathrm{Co}_{22} \mathrm{Pt}_{37}$. The compromise between magnetization and coercivity yields a nominal energy product maximum of $510 \mathrm{~kJ} / \mathrm{m}^{3}$ for $\mathrm{Fe}_{40} \mathrm{Co}_{22} \mathrm{Pt}_{38}$. ${ }^{6}$ The high magnetization of these structures results from the excess transition-metal content, which is beyond the ideal equiatomic composition of the $L 1_{0}$-ordered material.

A key requirement in nanostructured permanent magnetism is the right choice of materials, especially with the need to reduce the rare-earth content. ${ }^{7,8}$ In Ref. 7 , the optimum composition was obtained by maximizing the energy product in the limit of small soft inclusions. Interestingly, this procedure can be done fully analytically and yields the following explicit expression for the energy product of aligned hard-soft two-phase magnets

$$
(B H)_{\max }=\frac{K_{h} M s}{\Delta M}+\frac{2 K_{h}^{2}}{\mu_{o} \Delta M^{2}}-\frac{2 K_{H}^{2}}{\mu_{\circ} \Delta M^{2}} \sqrt{1+\frac{\mu_{o} M_{s} \Delta M}{K_{h}}}
$$




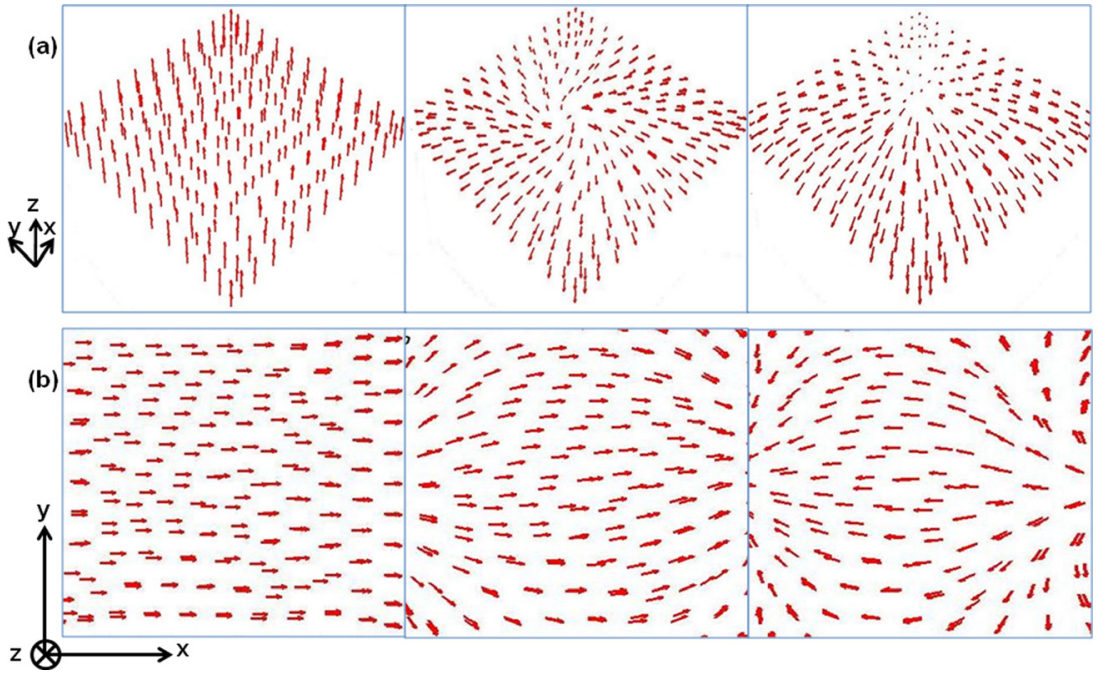

FIG. 1. (Color online) Calculated spin structure of the Fe layer in an $\mathrm{Fe} / \mathrm{FePt}$ nanocomposite: (a) magnetic field normal to the film plane (along $\mathrm{Z}$-axis) and (b) magnetic field in the film plane (along $\mathrm{x}$-axis). where $M_{\mathrm{s}}$ is the magnetization of the soft phase, $\Delta M=M_{\mathrm{s}}-M_{\mathrm{h}}$ the difference between the soft and hard magnetizations, and $K_{\mathrm{h}}$ the anisotropy of the hard phase. This equation has the functional structure $(B H)_{\max }=\mu_{\mathrm{o}} M_{\mathrm{s}}{ }^{2} \mathrm{f}(\eta)$, where the dimensionless ratio $\eta=\mu_{\mathrm{o}} M_{\mathrm{s}} \Delta M / K_{\mathrm{h}}$ can be considered as the small parameter of a hardness expansion:

$$
(B H)_{\max }=\frac{1}{4} \mu_{\mathrm{o}} M_{s}^{2}\left(1-\frac{1}{2} \eta+\frac{5}{16} \eta^{2}\right) .
$$

For a given soft phase, the energy product is therefore maximized by choosing a small ratio $\left(M_{\mathrm{s}}-M_{\mathrm{h}}\right) / K_{\mathrm{h}}$. This analysis shows that a hard phase of $\mathrm{Nd}_{2} \mathrm{Fe}_{14} \mathrm{~B}$ is better than $\operatorname{Pr}_{2} \mathrm{Fe}_{14} \mathrm{~B}$, in spite of the higher anisotropy field of $\operatorname{Pr}_{2} \mathrm{Fe}_{14} \mathrm{~B}$.

\section{HYSTERESIS-LOOP SHAPE}

In this section, we use numerical and analytical calculations to investigate the hysteresis-loop shape. Our model system consists of a soft layer on top of an aligned hard layer. Such structures can be produced, for example, by depositing iron onto an $L 1_{0}$-ordered hard-magnetic film with perpendicular anisotropy. ${ }^{6}$

To investigate the effect of the orientation of the magnetic field (perpendicular and in-plane) on the hysteresis loop and dynamics of the magnetization, we performed micromagnetic simulations using the Nmag software package. ${ }^{9}$ The system is modeled as a bilayer of FePt and Fe in a $50 \times 50 \times 22$ $\mathrm{nm}^{3}$ size cell with FePt layer acting as a hard phase of height $20 \mathrm{~nm}$ and $\mathrm{Fe}$ as the soft phase on the top of FePt of height 2 $\mathrm{nm}$. The magnetizations of $\mathrm{FePt}$ and $\mathrm{Fe}$ are taken as 1.138 $\mathrm{MA} / \mathrm{m}[1.43 \mathrm{~T}]$ and $1.711 \mathrm{MA} / \mathrm{m}$ [2.15 T], respectively. The assumed anisotropies are $6.6 \mathrm{MJ} / \mathrm{m}^{3}$ for FePt and zero for Fe.

Figures 1 and 2 shows the spin structures for the Fe part of the $\mathrm{Fe} / \mathrm{FePt}$ nanocomposite and the hysteresis loops for both field directions. In perpendicular fields, normal to the film plane and parallel to the $c$-axis, there is an abrupt drop of the soft phase's magnetization contribution at the softphase nucleation field $H_{n}$. This can also be seen from the spin structure shown in the Fig. 1(a). For fields in the film plane, the magnetization change is smooth and initially linear, meaning that the magnetization changes continuously, starting with the spins that are farthest away from the interface and forming a partial domain wall near the interface.

Analytically, we consider a field of the type $\mathbf{H}=H \cos \theta$ $\mathbf{e}_{\mathrm{z}}+H \sin \theta \mathbf{e}_{\mathrm{x}}$ and assume that the in-plane magnetization component $M_{\mathrm{x}}=M_{\mathrm{s}} \sin \phi$ of the soft phase is small, that is, $\mathrm{M}_{\mathrm{z}}=\mathrm{M}_{\mathrm{s}} \phi(\mathbf{r})$. Neglecting magnetostatic selfinteractions, the micromagnetic energy functional is then

$$
\mathrm{E}=\int\left(A(\nabla \phi)^{2}+\frac{\mu o M s H}{2} \cos \theta \phi^{2}(\mathbf{r})-\mu o M s H \sin \theta \phi(\mathbf{r})\right) \mathrm{dV} .
$$

The eigenmodes of this functional are well-known for number of geometries. They yield the nucleation field $H_{\mathrm{n}}(\theta=0)$ and serve as the starting point for lowest-order perturbation theory to determine the initial slope $\chi_{\mathrm{s}}$ of the in-plane loop $\left(\theta=90^{\circ}\right)$. For large soft inclusion, the modes all have a maximum $\phi(0)=\phi_{\mathrm{o}}$ in the center of the soft inclusion and $\phi=0$ at the hard-soft interface. For plate-like soft inclusions, the magnetization profile is $\phi(z)=\phi_{\mathrm{o}} \cos (\pi z / t)$; for cylindrical inclusions of radius $R$, the radial dependence is given by the Bessel function $\phi_{\mathrm{o}} J_{\mathrm{o}}(r / R)$, and for spherical inclusions, the mode is $2 R \phi_{\mathrm{o}} \sin (\pi r / 2 R) / \pi r$. The last function is basically the spherical Bessel function $j_{0}(x)$.

The initial susceptibility of the soft phase $\chi_{\mathrm{s}}=R^{2} / c_{\mathrm{o}}{ }^{2} l_{\mathrm{o}}{ }^{2}$, where $l_{\mathrm{o}}=\sqrt{A / \mu_{o} M_{s}^{2}}$ is the proper exchange length and

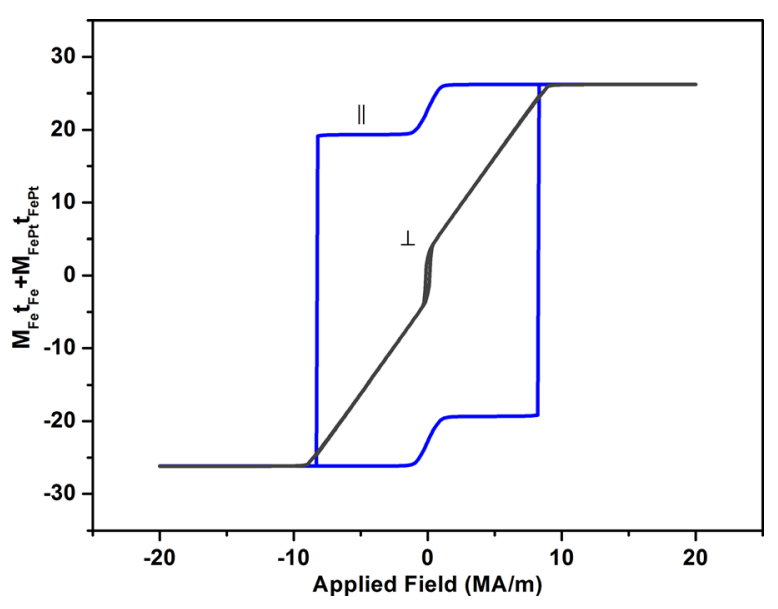

FIG. 2. (Color online) Hysteresis loops belonging to Fig. 1. 


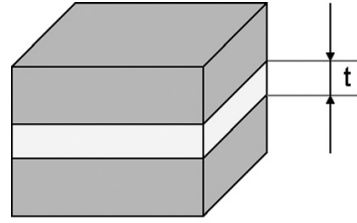

(a)

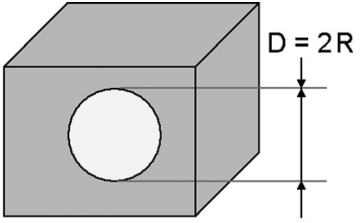

(b)
FIG. 3. Different hard-soft geometries: (a) embedded soft layer in a hard matrix and (b) embedded soft sphere.

$c_{\mathrm{o}}$ depends on the geometry of the inclusion. In particular, spherical inclusions are characterized by $c_{\mathrm{o}}=5.698$, platelike soft regions have $c_{\mathrm{o}}=2.467$, and for cylindrical inclusions (columns normal to the film plane) $c_{\mathrm{o}} \approx 4.1$. $^{2}$ Note that embedded plates or films of thickness $t$ have $R=t / 2$, whereas soft patches on a hard surface are characterized by $R=t$.

\section{INFLUENCE OF GEOMETRY}

It is well-established that the soft phase of a two-phase system should not be much larger than twice the domainwall width of the hard phase. This was initially deduced for layered systems, ${ }^{10}$ but it is also true for three-dimensional systems. ${ }^{7}$ However, there are also differences, both quantitatively and qualitatively. The corresponding dimensionality problem is related to the different localization behavior of the eigenmodes in one, two and three dimensions. ${ }^{11}$ Figure 3 shows two of the considered geometries.

The nucleation field of the soft phase is obtained by minimization of Eq. (3):

$$
H_{\mathrm{n}}=2 M_{\mathrm{s}} x_{\mathrm{o}}^{2} l_{\mathrm{o}}^{2} / R^{2} .
$$

Here $x_{\mathrm{o}}$ is the first zero of the eigenmode of Sec. III, that is, $x_{\mathrm{o}}=\pi$ for the spherical Bessel function (spheres), $x_{\mathrm{o}}=\pi / 2$ for $\cos (x)$ (embedded plates), and $x_{\mathrm{o}}=2.4048$ for Bessel function (cylinders). Explicitly, the ratio $H_{\mathrm{n}} / M_{\mathrm{s}}$ is equal to $19.74 l_{\mathrm{o}}{ }^{2} / R^{2}$ (spheres), $11.57 l_{\mathrm{o}}{ }^{2} / R^{2}$ (cylinders), $19.74 l_{\mathrm{o}}{ }^{2} / t^{2}$ (embedded plates), and $4.94 l_{\mathrm{o}}{ }^{2} / t^{2}$ (flat patches on hard surface). Figure 4 shows the coercivity $H_{\mathrm{n}}$ for a number of soft phases. The curves describe large soft inclusions ( $t$ or $D$ much larger than the domain-wall width of the hard phase). Specifically, the approach breaks down as $H_{\mathrm{n}}$ approaches the

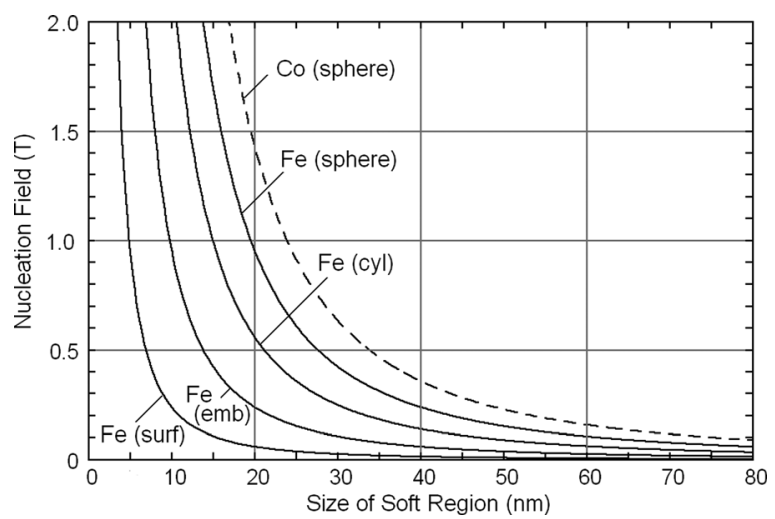

FIG. 4. Soft-phase nucleation field as a function of the size $2 R$ of the inclusion. For spherical soft inclusions (sphere) and cylindrical soft inclusions (cyl), $R$ is the radius of the inclusion, whereas for embedded (emb) and free surface (surf) soft layers, $2 R=t$. anisotropy field of the hard phase (Sec. V). Furthermore, the calculation does not include magnetostatic interactions.

From Fig. 4 we see that spheres have a much more forgiving size dependence of the switching field, corresponding to a factor 4 in coercivity. By analyzing the boundary condition at free surfaces, it can also be shown that free soft films (not capped by a hard layer) on an aligned hard substrate yield a factor 0.25 , that is, their coercivity is 4 times smaller than that of soft films embedded in hard matrix as shown in Fig. 3(a).

\section{DISCUSSION AND CONCLUSIONS}

These results confirms the original argument ${ }^{7,10}$ that the soft phase cannot be much larger than twice the domain-wall width $\delta_{\mathrm{B}}$ of the hard phase, but it also provides a differentiated and geometry-dependent answer to the question of how to define "much larger." Aside from these quantitative changes, there are also qualitative differences as $R$ gets smaller than $\delta_{\mathrm{B}}$ and $H_{\mathrm{n}}$ approaches $H_{\mathrm{o}}=2 K_{\mathrm{h}} / \mu_{\mathrm{o}} M_{\mathrm{h}}$. Layered magnetic structures are one-dimensional and undergo micromagnetic localization, as one can see, for example, by perturbation theory. ${ }^{11}$ The localization is accompanied by a slight reduction of the nucleation field, which obeys $\mathrm{d} H_{\mathrm{n}} / \mathrm{d} R=0$ at $R=0$ but deviates parabolically from $H_{\mathrm{o}}$ for $R>0$. This parabolic correction has been interpreted as a general feature of hard-soft nanostructures $^{12}$ but is, in fact, a one-dimensional localization phenomenon. The two-dimensional case (embedded soft cylinders) is marginal, with logarithmic rather than power-law corrections, and the three-dimensional case (embedded spheres) shows a fully developed plateau $H_{\mathrm{n}}(R)$ for small $R$.

In conclusion, we have analyzed how feature size and geometry affect the hard-magnetic performance of nanoscale permanent magnets. We advocate the use of the energy product as the key figure of merit for thin-film nanostructures, in spite of the fact that magnet volume and mass are much less important than in the bulk. Magnetic nanocomposites exhibit a rich physics as a function of geometry, feature size, field direction, and composition. One example is the localization behavior of the nucleation modes in layered structures.

\section{ACKNOWLEDGMENTS}

This research is supported by DST (DYNAMAG and Nano Mission, PKS, PM, AK), NSF MRSEC (PKS), ARO (SHL), BREM (RZ), ARPA-E (RS), DOE (DJS, GCH), and NCMN.

${ }^{1}$ R. M. Bozorth, Ferromagnetism (van Nostrand, Princeton, NJ, 1951).

${ }^{2}$ R. Skomski and J. M. D. Coey, Permanent Magnetism (Institute of Physics, Bristol, UK, 1999).

${ }^{3}$ R. Skomski, Y. Liu, J. E. Shield, G. C. Hadjipanayis, and D. J. Sellmyer, J. Appl. Phys. 107, 09A739 (2010).

${ }^{4}$ E. Jäger and R. Perthel, Magnetische Eigenschaften von Festkörpern (Akademie-Verlag, Berlin, 1983).

${ }^{5}$ T. Shima, K. Takanashi, Y. K. Takahashi, and K. Hono, Appl. Phys. Lett. 85, 2571 (2004).

${ }^{6}$ Y. Liu, T. A. George, R. Skomski, and D. J. Sellmyer, Appl. Phys. Lett. 99, 172504 (2011).

${ }^{7}$ R. Skomski and J. M. D. Coey, Phys. Rev. B 48, 15812 (1993).

${ }^{8}$ N. Jones, Nature 472, 22 (2011).

${ }^{9}$ T. Fischbacher, M. Franchin, G. Bordignon, H. Fangohr, IEEE Trans. Magn. 43, 2896 (2007).

${ }^{10}$ E. F. Kneller, IEEE Trans. Magn. 27, 3588 (1991).

${ }^{11}$ R. Skomski, J. Appl. Phys. 83, 6503 (1998).

${ }^{12}$ G. P. Zhao and X. L. Wang, Phys. Rev. B 74, 012409 (2006). 